\title{
Spindle assembly checkpoint-related meiotic defect in oocytes from LT/Sv mice has cytoplasmic origin and diminishes in older females
}

\author{
Steffen Hoffmann ${ }^{1}$, Marzena Król ${ }^{2}$ and Zbigniew Polanski ${ }^{1,2}$ \\ ${ }^{1}$ Department of Developmental Biology, Max Planck Institute of Immunobiology, Stuebeweg 51, D-79108 Freiburg, \\ Germany and ${ }^{2}$ Department of Genetics and Evolution, Institute of Zoology, Jagiellonian University, ul. Gronostajowa \\ 9, 30-387 Krakow, Poland
}

Correspondence should be addressed to Z Polanski at Department of Genetics and Evolution, Institute of Zoology, Jagiellonian University; Email: zbigniew.polanski@uj.edu.pl

S Hoffmann is now at ProQinase GmbH, Breisacher Strasse 117, 79106 Freiburg, Germany

\begin{abstract}
The spindle assembly checkpoint (SAC) ensures proper segregation of chromosomes by delaying anaphase onset until all kinetochores are properly attached to the spindle microtubules. Oocytes from the mouse strain LT/Sv arrest at the first meiotic metaphase (MI) due to, as reported recently, enormously prolonged activity of the SAC. We compared the dynamics of cyclin B1-GFP degradation, the process which is a measure of the SAC activity, in chromosomal and achromosomal halves of LT/Sv oocytes. In chromosome-containing oocyte halves arrested at MI, cyclin B1-GFP was not degraded indicating active SAC. However, in the halves lacking chromosomes, which is a condition precluding the SAC function, degradation always occurred confirming that MI arrest in LT/Sv oocytes is SAC dependent. Transferring the germinal vesicle (GV) from LT/Sv oocytes into the enucleated oocytes from wild-type mice resulted in the progression through meiosis one, indicating that a SAC-activating defect in LT/Sv oocytes is cytoplasmic, yet can be rescued by foreign cytoplasm. These results may help to define the etiology of the human infertility related to the oocyte $\mathrm{MI}$ arrest, indicating the involvement of the SAC as likely candidate, and point to GV transfer as the possible therapy. Finally, we found that majority of oocytes isolated from old LT/Sv mice complete the first meiosis. Reciprocal transfers of the GV between the oocytes from young and old LT/Sv females suggest that the factor(s) responsible for the reversal of the phenotype in oocytes from old mice is located both in the GV and in the cytoplasm. Reproduction (2012) 144 331-338
\end{abstract}

\section{Introduction}

The final stage of oogenesis, meiotic maturation, comprises a set of events crucial for the process of fertilization and embryonic development. During this period, the oocyte reduces the number of chromosomes by progressing from the prophase of the first meiotic cell cycle to the metaphase of the second meiotic cell cycle. The complex control of this transition (Kubiak et al. 2008) includes the activity of the spindle assembly checkpoint (SAC), a mechanism that enables errorless segregation of the chromosomes in dividing cells by blocking the onset of anaphase when sensing the spindle or chromosome anomalies (Musacchio \& Salmon 2007). The central element of the SAC pathway is monitoring the attachments of the microtubules to kinetochores even one unattached kinetochore generates the signal inhibiting anaphase (Rieder et al. 1994, 1995). The functionality of the SAC was recently shown also during both the first (Tsurumi et al. 2004, Homer et al. 2005a, 2005b) and the second (Tsurumi et al. 2004) meiotic division in mammalian oocytes. However, the high incidence of chromosome segregation errors in mammalian female meiosis raises the question on the lower effectiveness of the SAC in oocytes (Vogt et al. 2008, Holt \& Jones 2009).

The mouse mutant inbred strain LT/Sv exhibits a complex aberrant reproduction-related phenotype (Ciemerych et al. 2009). One of its features is the so-called oocyte metaphase I (MI) arrest as the majority of oocytes from LT/Sv strain instead finishing meiotic maturation stop their meiotic progression at the metaphase of the first meiotic division (Eppig 1978, Kaufman \& Howlett 1986, Maleszewski \& Yanagimachi 1995, Ciemerych \& Kubiak 1998). Recent work revealed that $\mathrm{MI}$ arrest of LT/Sv oocytes is caused by the inability to timely inactivate the SAC (Hupalowska et al. 2008). Moreover, prolonged SAC activity also occurs during mitotic divisions of the early LT/Sv embryos contributing, together with meiotic defect, to the low reproductive 
performance of this strain (Maciejewska et al. 2009). Several studies documented that Ml arrest in the oocytes may also underlie some cases of human infertility (Eichenlaub-Ritter et al. 1995, Harrison et al. 2000, Bergère et al. 2001, Windt et al. 2001, Levran et al. 2002, Schmiady \& Neitzel 2002, Combelles et al. 2003, Heindryckx et al. 2011). Therefore, LT/Sv oocytes may provide a valuable animal model to elucidate the etiology of similar disorders in humans and develop possible treatments.

As a consequence of the $\mathrm{Ml}$ arrest, the LT/Sv mice ovulate the oocytes at this stage which, when fertilized, give rise to the triploid digynic embryos. Interestingly, the study by Speirs \& Kaufman (1990) reported that the frequency of triploid embryos observed in LT/Sv females decreases with the age of the mothers. These authors suggested that this phenomenon may result from decreased frequency of $\mathrm{MI}$ arrest in LT/Sv oocytes in the older females; however, according to our knowledge, this hypothesis was never tested.

In this study, we analyzed the dynamics of cyclin B1 degradation, a process that is initiated only upon SAC inactivation and thus is an excellent way to study the status of the SAC activity (e.g. Clute \& Pines 1999, Tsurumi et al. 2004, Homer et al. 2005a, 2005b). The analysis was performed in the chromosome-containing and chromosome-devoid fragments of oocytes from $\mathrm{LT} / \mathrm{S} v$ mice. We show that cyclin B1 degradation is inhibited in most cases when the chromosomes are present. However, in the absence of chromosomes, which is a condition precluding the SAC activity, the cyclin B1 degradation proceeds effectively, thus delivering another proof that $\mathrm{MI}$ arrest in LT/Sv oocytes results from enormously prolonged SAC activity. Nuclear transfer experiments involving LT/Sv oocytes and oocytes from wild-type mice show that the defect causing the prolonged activity of the SAC in the LT/SV oocytes resides in the cytoplasm, not in the nucleus, of the prophase oocyte. Finally, we demonstrate that the frequency of $\mathrm{Ml}$ arrest in LT/Sv oocytes drops significantly in the older females. This last finding is consistent with the report by Speirs \& Kaufman (1990) who observed that the frequency of producing triploid embryos decreases in the old LT/Sv females.

\section{Results}

\section{Dynamics of cyclin B1-GFP degradation proves the involvement of the $S A C$ in the meiotic phenotype of $L T / S v$ oocytes}

Recently, we have shown that interference with the Bub1 function greatly improves the rate of the progression through the first meiosis of LT/Sv oocytes, which shows that $\mathrm{Ml}$ arrest is caused through sustained activity of the SAC (Hupalowska et al. 2008). To inquire further into the involvement of SAC in the meiotic phenotype of LT/SV mice, we performed the live-imaging of cyclin B1-GFP as the SAC activity inhibits cyclin B1 degradation (e.g. Clute \& Pines 1999, Tsurumi et al. 2004, Homer et al. 2005a, 2005b). The oocytes were bisected to produce the chromosome-containing and the chromosome-devoid halves. Assuming that the LT/Sv defect is indeed related to the SAC activity, the cyclin B1-GFP level should be stabilized in the halves containing the chromosomes. In contrast, the degradation of cyclin B1-GFP should proceed in the chromosome-lacking oocyte halves due to the absence of the kinetochores, which provide the crucial element of the SAC pathway. In two experiments, the live imaging of cyclin B1-GFP was performed in eight chromosome-containing and seven chromosome-lacking halves as well as in three intact whole oocytes.

Among chromosome-containing halves, only two out of eight extruded the first polar body (PB1), which is consistent with the phenotype of LT/Sv oocytes. PB1 extrusion was accompanied with the rapid degradation of cyclin B1-GFP (Fig. 1), thus showing profile of cyclin B1 degradation typical for the wild-type oocytes undergoing the first meiotic division (Ledan et al. 2001, Homer et al. 2005a, 2005b). In the rest of chromosomecontaining halves (six out of eight), PB1 extrusion did not occur, which indicated MI arrest. In such oocytes, cyclin B1-GFP showed unusual profile lacking rapid degradation but revealing rather the stabilization of the protein at the high level (Fig. 1). Accordingly, the cyclin B1-GFP degradation did not occur also in the whole (non-bisected) oocytes arrested at MI (three oocytes, data not shown). In contrast, in all chromosome-devoid oocyte halves $(n=7)$, cyclin B1 was degraded rapidly (Fig. $1 \mathrm{~A}$ and $\mathrm{B}$ ). We have recently shown that degradation of cyclin B1-GFP in the chromosomedevoid oocyte halves is inhibited in the presence of MG132, which indicates that such degradation is proteasome dependent (Hoffmann et al. 2011). The difference between the behavior of the chromosomecontaining halves (stabilization of cyclin B1-GFP) and the chromosome-devoid halves (rapid cyclin B1-GFP degradation) was statistically significant. These results confirm the involvement of the SAC in MI arrest in LT/SV oocytes. The time of cyclin B1-GFP degradation in chromosome-devoid halves was earlier than in the chromosome-containing halves, which extruded PB1 (Fig. 1B). This results from the SAC activity maintained in the latter until the kinetochore-microtubule attachments are established.

\section{The $M I$ arrest in $L T / S v$ oocytes originates from a cytoplasmic defect}

To inquire whether the primary defect, which prolongs SAC activity in LT/Sv oocytes, has cytoplasmic or nuclear origin, we performed the set of germinal vesicle (GV) transfer experiments. The GVs removed from LT/SV 


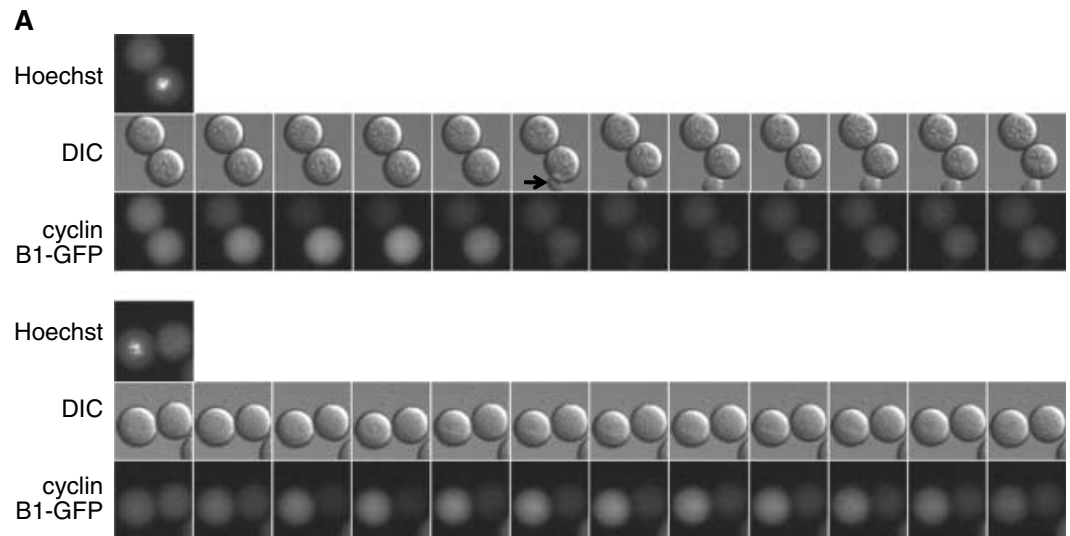

B

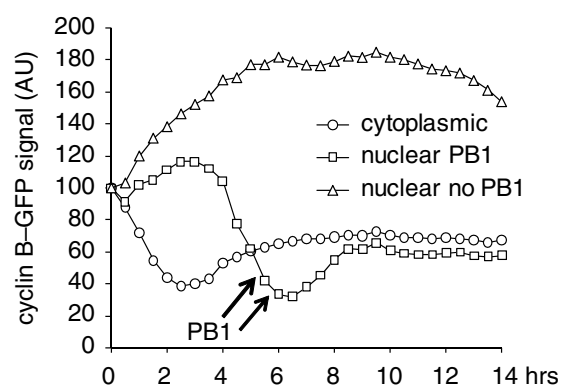

oocytes were transferred into enucleated GV stage OF1 oocytes and vice versa. Almost all oocytes that were manipulated to combine LT/Sv nucleus with OF1 cytoplasm extruded PB1. On the other hand, the majority of oocytes created by the transfer of wild-type GV into LT/Sv cytoplasm displayed arrest at the MI typical for LT/Sv oocytes (Table 1). These experiments clearly show that the defect underlying the MI arrest in LT/Sv oocytes has cytoplasmic origin.

\section{The incidence of the oocyte $\mathrm{MI}$ arrest is greatly reduced in older LT/SV females}

We tested the meiotic progression in vitro in oocytes isolated from the LT/Sv females of different age. Whereas in the females up to 28 weeks old, only $23-31 \%$ of oocytes completed the first meiotic division; the proportion of such oocytes increased significantly in older females reaching $65 \%$ at the age $47-52$ weeks (Fig. 2).

To see whether cytoplasm from a young oocyte can reestablish the $\mathrm{Ml}$ arrest with an older nucleus (or vice versa), we have performed reciprocal GV transfer between LT/Sv oocytes isolated from young (age 8-22 weeks) or old (44-50 weeks) females (Table 2). The overall frequency of the oocytes that extruded PB1 was reduced in these conditions (compare, for e.g. Fig. 2 for the rate of PB1 extrusion in intact LT/Sv oocytes), nevertheless the difference between control young/ young hybrid oocytes (composed of young GV and
Figure 1 Imaging of the cyclin B1-GFP in the halves of LT/Sv oocytes undergoing meiotic maturation. (A) Fluorescence and DIC images. The upper and the lower panel show examples of the pairs of the oocyte halves obtained from the bisection of a single oocyte. Note that nuclear half (indicated by Hoechst staining) shown on the upper panel extruded PB1 (black arrow), whereas the nuclear half shown on the lower panel was arrested at MI. The images in $1 \mathrm{~h}$ intervals are shown starting at 15 th min of imaging. (B) Semiquantitative analysis of the cyclin B1-GFP fluorescence signal. The mean values are shown for nuclear halves, which extruded PB1 (timing indicated by arrows; $n=2$ ), nuclear halves, which arrested at $\mathrm{MI}(n=6)$, and cytoplasmic halves $(n=7)$. The data were collected in two independent experiments. The difference between the behavior of the chromosome-deprived halves (rapid cyclin B1-GFP degradation) and the chromosomecontaining halves (stabilization of cyclin B1-GFP) is statistically significant $(P<0.05$; one-tailed Fisher exact probability test). young cytoplasm) and old/old oocytes (old GV and old cytoplasm) was retained and close to the statistical significance ( $P=0.088$; Table 2$)$. On the other hand, in the young/old hybrids as well as in the old/young ones, the proportion of PB1 extrusion was similar to that in the old/old oocytes (Table 2). This suggests that the factor(s) responsible for the increased rate of the PB1 extrusion in the oocytes from older mice is located both in their nucleus and in their cytoplasm.

\section{Discussion}

The inability to complete the first meiotic division by the majority of oocytes from the strain LT/Sv was recently attributed to the prolonged activity of the SAC (Hupalowska et al. 2008). In this study, the involvement of SAC in this specific meiotic phenotype was further confirmed by imaging cyclin B1-GFP. As previously shown in mouse oocytes undergoing meiosis I/meiosis II transition, the cyclin B1 degradation is inhibited until

Table 1 The progression through the first meiotic division in the hybrid oocytes produced by germinal vesicle transfer.

\begin{tabular}{lcc}
\hline $\begin{array}{l}\text { Composition of } \\
\text { hybrid oocyte } \\
\text { (GV:cytoplasm) }\end{array}$ & $\begin{array}{c}\text { No. of } \\
\text { oocytes }\end{array}$ & $\begin{array}{c}\text { No. of oocytes } \\
\text { extruding PB1 }(\%)\end{array}$ \\
\hline LT/Sv:OF1 & 33 & $24(72.7)^{\mathrm{a}}$ \\
OF1:LT/Sv & 26 & $8(30.8)^{\mathrm{a}}$
\end{tabular}

${ }^{\mathrm{a}}$ Frequency of oocytes extruding PB1 differs significantly between groups; $P<0.005\left(2 \times 2 \chi^{2}\right.$ test with Yates correction). 


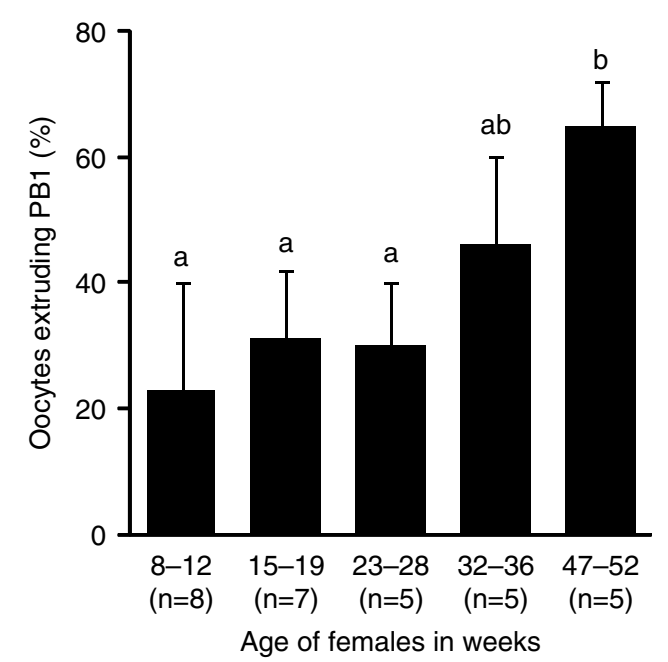

Figure 2 Oocytes from older LT/Sv females complete meiosis with higher efficiency. Bars represent mean \pm s.E.M. Different letters indicate significant difference ( $P<0.05$; ANOVA with Bonferroni post hoc test). The number of females is shown in the parentheses. Mean numbers of tested oocytes/female in different age groups were as follows: 8-12 weeks, 15.9 oocytes (range $8-22)$; 15-19 weeks, 17.3 (12-32); 23-28 weeks, 17.2 (9-26); 32-36 weeks, 14.2 (9-21); and 47-52 weeks, 10.8 (6-19).

the SAC remains active (Tsurumi et al. 2004, Homer et al. 2005a, 2005b). Accordingly, in the few LT/Sv oocytes that complete first meiosis, the degradation of cyclin B1 occurs with similar timing and similar profile as in the typical mouse oocytes coinciding with the PB1 extrusion. On the other hand, in oocytes that were unable to undergo the first meiotic division, we never observed rapid and profound degradation of cyclin B1-GFP. Instead, the level of cyclin B1-GFP in such oocytes remained stabilized at the high level. This result is consistent with earlier, western blot-derived observation on the accumulation of cyclin B in the Ml-arrested LT/Sv oocytes (Hampl \& Eppig 1995). We show, however, that the removal of the chromosomes from the LT/Sv oocytes (by producing the oocyte halves lacking the chromosomes) invariably resulted with the rapid and efficient degradation of cyclin B1-GFP. As the kinetochores provide the crucial element of the SAC pathway, the removal of chromosomes (along with the kinetochores) breaks down the SAC, thus initiating cyclin B1 degradation. Efficient degradation of cyclin B1 occurring in LT/Sv oocytes upon chromosome removal provides clear evidence that $\mathrm{MI}$ arrest is mediated by the activity of SAC supporting our previous report (Hupalowska et al. 2008). These results also show that the proteolytic mechanism that acts downstream of the SAC and executes cyclin B1 degradation functions efficiently in LT/Sv oocytes.

The nature of the SAC-affecting defect in LT/Sv oocytes remained unclear until now. Using nuclear transfer, we show that the hybrid oocytes composed of LT/SV GV and wild-type cytoplasm do not suffer from the MI arrest whereas the hybrid oocytes containing wild-type GV within the LT/Sv cytoplasm do arrest at MI. These results show that the defect causing prolonged SAC activity in $\mathrm{LT} / \mathrm{Sv}$ oocytes is cytoplasmic. Assuming that the prolonged checkpoint activity in LT/Sv oocytes reflects SAC-sensed unidentified anomalies within the MI spindle, these results suggest that the primary cause of the $\mathrm{MI}$ arrest may lie in the spindle components recruited from the cytoplasm. In this context, it is noticeable that LT/Sv oocytes were reported to exhibit unusual organization of centrosomes at the prophase stage (Albertini \& Eppig 1995). On the other hand, previous studies did not reveal abnormal morphology of the $\mathrm{MI}$ spindle in $\mathrm{LT} / \mathrm{SV}$ oocytes $7 \mathrm{~h}$ after GV breakdown (GVBD; Ciemerych \& Kubiak 1998), i.e. at the time when oocytes from other mouse strains already extrude the PB1s or are close to start this process (Polanski 1997). Therefore, another possibility explaining LT/Sv phenotype shall assume that spindle formation in LT/Sv oocytes is unperturbed and the $\mathrm{MI}$ arrest results from the lesion within the SAC pathway itself. According to our results, future research exploring this possibility should concentrate on the putative SAC components, which were proven to reside in the cytoplasm rather than nucleus of the prophase oocyte.

Several recent studies showed that some cases of unexplained infertility in humans result from arrest of the oocytes at the MI stage (Eichenlaub-Ritter et al. 1995, Harrison et al. 2000, Bergère et al. 2001, Windt et al. 2001, Levran et al. 2002, Schmiady \& Neitzel 2002, Combelles et al. 2003, Heindryckx et al. 2011). It is obvious that the SAC represent a potent mechanism arresting the cell at metaphase. A number of factors may affect the spindle structure, thus keeping the SAC active; moreover, the prolonged SAC activity may reflect a defect in the SAC pathway itself. Thus, a lot of scenarios may lead to maintain the SAC active resulting in the metaphase arrest. Therefore, the SAC activity, which was shown to block oocytes at MI in LT/Sv mice (Hupalowska et al. 2008; this study), shall be considered as major candidate responsible for the cases of $\mathrm{MI}$ arrest also in human oocytes. The cases of MII arrest in fertilized human eggs were also reported (Levran et al. 2002). These may result from defective sperm being unable to

Table 2 The progression through the first meiotic division in the hybrid LT/Sv oocytes produced by germinal vesicle transfer between oocytes from young and old females.

\begin{tabular}{lcc}
\hline $\begin{array}{l}\text { Composition of } \\
\text { hybrid LT/Sv oocyte } \\
\text { (GV:cytoplasm) }\end{array}$ & $\begin{array}{c}\text { No. of } \\
\text { oocytes }\end{array}$ & $\begin{array}{c}\text { No. of oocytes } \\
\text { extruding PB1 }(\%)\end{array}$ \\
\hline Young:old & 36 & $9(25 \%)$ \\
Old:young & 34 & $8(23.5 \%)$ \\
Old:old & 33 & $9(27.3 \%)$ \\
Young:young & 35 & $4(11.4 \%)$
\end{tabular}

The age of the young females was 8-22 weeks and the old ones 44-50 weeks. 
deliver activating stimulus or from oocyte defect being unable to respond properly to sperm penetration. Notably, as the SAC was shown to function in oocytes also during the second meiotic cycle (Tsurumi et al. 2004, Homer et al. 2005a), its involvement in at least some cases of human infertility related to the oocyte MII arrest cannot be excluded.

The GV transfer or the cytoplasm transfer represents the possible treatment of human infertility related to oocyte defect (Cohen et al. 1997, Krey et al. 2001), although the safety of these procedures still needs full confirmation (Levy et al. 2004). We show that transfer of GV from LT/Sv oocyte into the wild-type cytoplasm rescues the meiotic progression of LT/Sv nucleus. This points to GV transfer (or perhaps transfer of the donor cytoplasm from healthy oocyte into patient's oocyte) as a possible effective treatment of the cases of recurrent human oocyte $\mathrm{MI}$ arrest, especially in the light of unsuccessful attempts of an alternative therapy (Heindryckx et al. 2011).

We show dramatic decrease in the incidence of oocytes blocked by the SAC at MI in oocytes from aged $\mathrm{LT} / \mathrm{S} v$ females. This may mean that the primary cytoplasmic defect, which activates the SAC in LT/SV oocytes, disappears with the female age. Alternatively, it might be possible that in older females such primary defect persists, and the low frequency of MI arrest results, paradoxically, from decreased quality of the oocytes in older females, which is a well-known phenomenon. One could consider at least three plausible explanations linking such a maternal age-dependent phenotype of LT/Sv oocytes with age-dependent deterioration of oocytes. Some previous studies reported the decreased level of the SAC proteins or their transcripts in the oocytes or ovaries of older females in mice (Baker et al. 2004, Pan et al. 2008) and in humans (Steuerwald et al. 2001), suggesting the lower effectiveness of the SAC during mammalian female meiosis and thus implicating defective SAC as one of the possible sources of agerelated aneuploidy (Holt \& Jones 2009). Therefore, one could assume that the primary cytoplasmic defect persists in the oocytes from old LT/Sv females; however, the weakened SAC is unable to maintain the MI arrest. The weakness of such hypothesis is, however, that the mouse study provided no proof on the decreased SAC activity in the oocytes from old females (Duncan et al. 2009). Instead, several recent studies show a failure in the chromosome cohesion in the oocytes from old females, which becomes a leading hypothesis elucidating maternal age-related aneuploidy (Liu \& Keefe 2008, Chiang et al. 2010, 2011, Lister et al. 2010). Therefore, the second possible explanation implies that in oocytes from old LT/Sv females, the chromosomes lose their cohesion, which results in their separation and possibly induce the PB1 extrusion. Lastly, it is also possible that deterioration of the oocyte quality in the older females could affect the cytostatic factor (CSF) activity, which normally blocks the oocyte at the MII stage. Hirao \& Eppig (1997) previously demonstrated that the CSF activity is required for the maintenance of LT/Sv oocytes at the permanent $\mathrm{Ml}$ arrest. Consistently, any failure in the CSF activity, which could eventually occur in the oocytes from old LT/Sv females, would result in the breakdown of the $\mathrm{MI}$ arrest and completion of the first meiosis.

The experiments with the reciprocal transfer of GV between the oocytes isolated from LT/Sv females of different age give some indications on the nature of the change of the phenotype in oocytes from old LT/Sv mice (Table 2). As demonstrated in Table 2, control hybrids reconstituted from 'old' oocytes extrude PB1 with higher efficiency than the control hybrids reconstituted from 'young' oocytes. On the other hand, the mixed hybrids composed of young GV and old cytoplasm (young GV/ old cytoplasm) as well as the hybrids with reversed combination (old GV/young cytoplasm) have the maturation rate similar to the reconstituted 'old' oocytes. Thus, the factor responsible for the elevated frequency of PB1 extrusion of 'old' LT/Sv oocytes seems to be located both in the GV and in the cytoplasm. We, on the other hand, know that the primary defect responsible for the $\mathrm{MI}$ arrest in the young LT/Sv oocytes is purely cytoplasmic (Table 1). As mentioned earlier, one of the possible explanations of the change of the phenotype in old LT/SV oocytes comprises the retraction of this primary cytoplasmic defect responsible for $\mathrm{MI}$ arrest. If this primary defect, however, would retract in the 'old' oocytes, we should see the increase in PB1 extrusion only in the 'young GV/old cytoplasm' hybrids, but not in the 'old GV/young cytoplasm' ones. The obtained results, therefore, favor the alternative hypothesis that in the oocytes from old LT/Sv females, the primary defect still persists, but, due to some other changes in old oocytes, it cannot be expressed phenotypically. Moreover, we should also exclude the possibility that the elevated frequency of the PB1 extrusion in the 'old' oocytes could be related to the weakening of the cohesins as, in that case, we should see increased proportion of PB1 extrusion in the 'old GV/young cytoplasm' combination but not in the young GV/old cytoplasm' ones.

Therefore, from the possible explanations for the increased frequency of completion of the first meiosis in oocytes from old LT/Sv females presented earlier, we should consider as the most likely ones the two: that in the oocytes from old females either the SAC or the CSF weakens. Notably, the immunofluorescence studies revealed that crucial CSF component Emi2 (Shoji et al. 2006) is located in the GV of mouse oocytes (Suzuki et al. 2010) and the GV localization was demonstrated also for the key SAC proteins Mad2 (Wang et al. 2009), BubR1 (Wei et al. 2010) and Bub3 (Li et al. 2009). It is possible that these proteins are also located in the cytoplasm as even slightly elevated amount of the protein in the GV may give the distinct immunofluorescent signal when compared with the cytoplasm. 
We have shown such relationship previously for the PLK1, which gave distinct GV-associated staining; however, the western blots of GV-containing and GV-deprived oocyte halves revealed that it is also abundant in the cytoplasm (Pahlavan et al. 2000). Therefore, both CSF-related and the SAC-related explanations for the change of the phenotype in the old LT/SV oocytes seem to be at present equally possible.

\section{Materials and Methods}

\section{Animals}

The mice from LT/Sv strain as well as the wild-type mice from the stock OF1 were used. The experiments were approved by the Local Ethics Committee no. 1 in Krakow, Poland, and Regierungspraesidium in Freiburg, Germany, according to the European Union Council Directive 86/609/EEC of 24 November 1986.

\section{Oocyte collection, micromanipulation, and culture}

Mouse oocytes at the GV stage (GV oocytes) were isolated from the ovaries of 2- to 5-month-old females of OF1 or LT/Sv mice, except experiments designed to test the effect of age on the meiotic phenotype of LT/Sv mice in which the cohorts of mice at specified age were used. Oocytes were cultured as described previously (Polanski et al. 2005) in the M2 medium (Sigma-Aldrich) under mineral oil (Sigma-Aldrich). GV transfer was performed as described previously (Polanski et al. 2005) in the M2 medium supplemented with the dbcAMP (Sigma-Aldrich) to prevent meiosis resumption before finishing the micromanipulation procedure. Rinsing out dbcAMP was considered as the start of the culture of micromanipulated oocytes.

\section{Dynamics of cyclin B1-GFP degradation}

Oocytes from LT/Sv mice were cultured for $2 \mathrm{~h}$ and these which resumed meiosis (indicated by GVBD) were injected with cyclin B1-GFP RNA (Ledan et al. 2001). After $1 \mathrm{~h}$ of the culture, the zona pellucida was removed from injected oocytes by the pronase treatment (Polanski 1997) and oocytes were transferred into the cytochalasin B containing medium in which they were bisected into two halves as described previously (Tarkowski 1977, Hoffmann et al. 2006). To discriminate between chromosome-containing and chromosome-devoid oocyte halves, they were then labeled for $30 \mathrm{~min}$ at $37{ }^{\circ} \mathrm{C}$ with vital Hoechst (bisbenzimide, H 33258, Sigma-Aldrich, $1 \mu \mathrm{g} / \mathrm{ml}$ ) and finally cultured in the $\mathrm{M} 2$ medium on the stage of inverted Zeiss Axiovert200M microscope for live imaging. At the start of the culture, the image using the filter appropriate for vital Hoechst was taken to identify the halves containing the chromosomes and those which contained the cytoplasm only. During the further culture, the images were captured every 15 min at GFP and DIC channels. The intensity of the fluorescence generated by GFP was quantified on the acquired images using Adobe Photoshop. In each image analyzed, the virtual circular frame with the diameter slightly less than the diameter of the oocyte half was created. This frame was superimposed over the oocyte half and the mean luminosity of the marked area was measured using 'histogram' function. Then, the same frame was moved to cover the next oocyte half followed by measurement. Using the same frame, all oocyte halves on all the images within a whole single experiment were analyzed. The same frame was also superimposed over the area where no oocyte halves were present (always the left lower corner of the image) to measure the background on the each image. The background value was then subtracted from the value obtained for each oocyte half analyzed on a given image.

\section{The effect of female age on the meiotic progression of $L T / S V$ oocytes}

Oocytes were isolated in the $\mathrm{M} 2$ and after removing the cumulus cells placed in the culture for $18 \mathrm{~h}$. At the end of the culture, the number of oocytes that extruded the PB1 was determined.

\section{Statistical analysis}

The data were analyzed using $2 \times 2 \chi^{2}$ test (in some cases, the Fisher exact probability test was used as more appropriate for the analysis of samples with low numbers) or one-way ANOVA. In the latter case, the percentage values were first processed for angular arcsine transformation, and after running ANOVA, the differences between groups were determined using Bonferroni post hoc test. $P<0.05$ was considered statistically significant.

\section{Declaration of interest}

The authors declare that there is no conflict of interest that could be perceived as prejudicing the impartiality of the research reported.

\section{Funding}

This work was supported by Max Planck Society and the Polish National Science Centre (grant no. DEC-2011/01/B/NZ3/ 00190) both to Z Polanski.

\section{References}

Albertini DF \& Eppig JJ 1995 Unusual cytoskeletal and chromatin configurations in mouse oocytes that are atypical in meiotic progression. Developmental Genetics 16 13-19. (doi:10.1002/dvg.1020160105)

Baker DJ, Jeganathan KB, Cameron JD, Thompson M, Juneja S, Kopecka A, Kumar R, Jenkins RB, de Groen PC, Roche P et al. 2004 BubR1 insufficiency causes early onset of aging-associated phenotypes and infertility in mice. Nature Genetics 36 744-749. (doi:10.1038/ng1382)

Bergère $\mathbf{M}$, Lombroso $\mathbf{R}$, Gombault $\mathbf{M}$, Wainer $\mathbf{R}$ \& Selva J 2001 An idiopathic infertility with oocytes metaphase I maturation block: case report. Human Reproduction 16 2136-2138. (doi:10.1093/humrep/16. 10.2136)

Chiang T, Duncan FE, Schindler K, Schultz RM \& Lampson MA 2010 Evidence that weakened centromere cohesion is a leading cause of age-related aneuploidy in oocytes. Current Biology 20 1522-1528. (doi:10.1016/j.cub.2010.06.069) 
Chiang T, Schultz RM \& Lampson M 2011 Age-dependent susceptibility of chromosome cohesion to premature separase activation in mouse oocytes. Biology of Reproduction 85 1279-1283 (doi:10.1095/biolreprod.111.094094). (doi:10.1095/biolreprod.111.094094)

Ciemerych MA \& Kubiak JZ 1998 Cytostatic activity develops during meiosis I in oocytes of LT/Sv mice. Developmental Biology 200 198-211. (doi:10.1006/dbio.1998.8930)

Ciemerych MA, Archacka K, Polanski Z \& Kubiak JZ 2009 Cell cycle modification during oocyte maturation and early development of LT/SV mice: normal development or teratoma formation? In Cell Cycle and Development in Vertebrates, pp 181-202. Eds JZ Kubiak, MA Ciemerych \& L Richard-Parpaillon. Trivandrum, India: Research Signpost.

Cohen J, Scott R, Schimmel T, Levron J \& Willadsen S 1997 Birth of infant after transfer of anucleate donor oocyte cytoplasm into recipient eggs. Lancet 350 186-187. (doi:10.1016/S0140-6736(05)62353-7)

Combelles CM, Albertini DF \& Racowsky C 2003 Distinct microtubule and chromatin characteristics of human oocytes after failed in-vivo and in-vitro meiotic maturation. Human Reproduction 18 2124-2130. (doi:10.1093/humrep/deg419)

Clute P \& Pines J 1999 Temporal and spatial control of cyclin B1 destruction in metaphase. Nature Cell Biology 1 82-87. (doi:10.1038/10049)

Duncan FE, Chiang T, Schultz RM \& Lampson MA 2009 Evidence that a defective spindle assembly checkpoint is not the primary cause of maternal age-associated aneuploidy in mouse eggs. Biology of Reproduction 81 768-776. (doi:10.1095/biolreprod.109.077909)

Eichenlaub-Ritter U, Schmiady H, Kentenich H \& Soewarto D 1995 Recurrent failure in polar body formation and premature chromosome condensation in oocytes from a human patient: indicators of asynchrony in nuclear and cytoplasmic maturation. Human Reproduction $\mathbf{1 0}$ 2343-2349.

Eppig JJ 1978 Granulosa cell deficient follicles: occurrence, structure, and relationship to ovarian teratocarcinogenesis in strain LT/SV mice. Differentiation 12 111-120. (doi:10.1111/j.1432-0436.1979.tb00996.x)

Hampl A \& Eppig JJ 1995 Analysis of the mechanism(s) of metaphase I arrest in maturing mouse oocytes. Development 121 925-933.

Harrison K, Sherrin D \& Keeping J 2000 Repeated oocyte maturation block. Journal of Assisted Reproduction and Genetics 17 231-233. (doi:10.1023/A:1009401614028)

Heindryckx B, Lierman S, Combelles CM, Cuvelier CA, Gerris J \& De Sutter P 2011 Aberrant spindle structures responsible for recurrent human metaphase I oocyte arrest with attempts to induce meiosis artificially. Human Reproduction 26 791-800. (doi:10.1093/humrep/ deq400)

Hirao Y \& Eppig JJ 1997 Analysis of the mechanism(s) of metaphase I arrest in strain LT mouse oocytes: participation of MOS. Development 124 5107-5113.

Hoffmann S, Tsurumi C, Kubiak JZ \& Polanski Z 2006 Germinal vesicle material drives meiotic cell cycle of mouse oocyte through the $3^{\prime} \cup T R-$ dependent control of cyclin B1 synthesis. Developmental Biology 292 46-54. (doi:10.1016/j.ydbio.2005.12.052)

Hoffmann S, Maro B, Kubiak JZ \& Polanski Z 2011 A single bivalent efficiently inhibits cyclin B1 degradation and polar body extrusion in mouse oocytes indicating robust SAC during female meiosis I. PLOS ONE 6 e27143. (doi:10.1371/journal.pone.0027143)

Holt JE \& Jones KT 2009 Control of homologous chromosome division in the mammalian oocyte. Molecular Human Reproduction 15 139-147.

Homer HA, McDougall A, Levasseur M, Murdoch AP \& Herbert M 2005a Mad2 is required for inhibiting securin and cyclin B degradation following spindle depolymerisation in meiosis I mouse oocytes. Reproduction 130 829-843. (doi:10.1530/rep.1.00856)

Homer HA, McDougall A, Levasseur M, Yallop K, Murdoch AP \& Herbert M 2005b Mad2 prevents aneuploidy and premature proteolysis of cyclin B and securin during meiosis I in mouse oocytes. Genes and Development 19 202-207. (doi:10.1101/gad.328105)

Hupalowska A, Kalaszczynska I, Hoffmann S, Tsurumi C, Kubiak JZ, Polanski Z \& Ciemerych MA 2008 Metaphase I arrest in LT/Sv mouse oocytes involves the spindle assembly checkpoint. Biology of Reproduction 79 1102-1110. (doi:10.1095/biolreprod.108.069609)

Kaufman MH \& Howlett SK 1986 The ovulation and activation of primary and secondary oocytes in LT/Sv strain mice. Gamete Research $\mathbf{1 4}$ 255-264. (doi:10.1002/mrd.1120140309)
Krey L, Liu H, Zhang J \& Grifo J 2001 Fertility and maternal age strategies to improve pregnancy outcome. Annals of the New York Academy of Sciences 943 26-33. (doi:10.1111/j.1749-6632.2001.tb03787.x)

Kubiak JZ, Ciemerych MA, Hupalowska A, Sikora-Polaczek M \& Polanski Z 2008 On the transition from the meiotic to mitotic cell cycle during early mouse development. International Journal of Developmental Biology 52 201-217. (doi:10.1387/ijdb.072337jk)

Ledan E, Polanski Z, Terret ME \& Maro B 2001 Meiotic maturation of the mouse oocyte requires an equilibrium between cyclin B synthesis and degradation. Developmental Biology 232 400-413. (doi:10.1006/dbio. 2001.0188)

Levran D, Farhi J, Nahum H, Glezerman M \& Weissman A 2002 Maturation arrest of human oocytes as a cause of infertility: case report. Human Reproduction 17 1604-1609. (doi:10.1093/humrep/17.6.1604)

Levy R, Elder K \& Ménézo Y 2004 Cytoplasmic transfer in oocytes: biochemical aspects. Human Reproduction Update 10 241-250. (doi:10.1093/humupd/dmh016)

Li M, Li S, Yuan J, Wang ZB, Sun SC, Schatten H \& Sun QY 2009 Bub3 is a spindle assembly checkpoint protein regulating chromosome segregation during mouse oocyte meiosis. PLOS ONE 4 e7701. (doi:10.1371/ journal.pone.0007701)

Lister LM, Kouznetsova A, Hyslop LA, Kalleas D, Pace SL, Barel JC, Nathan A, Floros V, Adelfalk C, Watanabe Y et al. 2010 Age-related meiotic segregation errors in mammalian oocytes are preceded by depletion of cohesin and Sgo2. Current Biology 20 1511-1521. (doi:10.1016/j.cub.2010.08.023)

Liu L \& Keefe DL 2008 Defective cohesin is associated with age-dependent misaligned chromosomes in oocytes. Reproductive Biomedicine Online 16 103-112. (doi:10.1016/S1472-6483(10)60562-7)

Maciejewska Z, Polanski Z, Kisiel K, Kubiak JZ \& Ciemerych MA 2009 Spindle assembly checkpoint-related failure perturbs early embryonic divisions and reduces reproductive performance of $\mathrm{LT} / \mathrm{Sv}$ mice. Reproduction 137 931-942. (doi:10.1530/REP-09-0011)

Maleszewski M \& Yanagimachi H 1995 Spontaneous and sperm-induced activation of oocytes in LT/Sv strain mice. Development, Growth \& Differentiation 37 679-685. (doi:10.1046/j.1440-169X.1995.t01-5-00006.x)

Musacchio A \& Salmon ED 2007 The spindle-assembly checkpoint in space and time. Nature Reviews. Molecular Cell Biology 8 379-393. (doi:10.1038/nrm2163)

Pahlavan G, Polanski Z, Kalab P, Golsteyn R, Nigg EA \& Maro B 2000 Characterization of polo-like kinase 1 during meiotic maturation of the mouse oocyte. Developmental Biology 220 392-400. (doi:10.1006/dbio.2000.9656)

Pan H, Ma P, Zhu W \& Schultz RM 2008 Age-associated increase in aneuploidy and changes in gene expression in mouse eggs. Developmental Biology 316 397-407. (doi:10.1016/j.ydbio.2008.01.048)

Polanski Z 1997 Strain difference in the timing of meiosis resumption in mouse oocytes: involvement of a cytoplasmic factor(s) acting presumably upstream of the dephosphorylation of p34(cdc2) kinase. Zygote 5 105-109. (doi:10.1017/S0967199400003774)

Polanski Z, Hoffmann S \& Tsurumi C 2005 Oocyte nucleus controls progression through meiotic maturation. Developmental Biology 281 184-195. (doi:10.1016/j.ydbio.2005.02.024)

Rieder CL, Schultz A, Cole R \& Sluder G 1994 Anaphase onset in vertebrate somatic cells is controlled by a checkpoint that monitors sister kinetochore attachment to the spindle. Journal of Cell Biology 127 1301-1310. (doi:10.1083/jcb.127.5.1301)

Rieder CL, Cole RW, Khodjakov A \& Sluder G 1995 The checkpoint delaying anaphase in response to chromosome monoorientation is mediated by an inhibitory signal produced by unattached kinetochores. Journal of Cell Biology 130 941-948. (doi:10.1083/jcb.130.4.941)

Schmiady H \& Neitzel H 2002 Arrest of human oocytes during meiosis I in two sisters of consanguineous parents: first evidence for an autosomal recessive trait in human infertility: case report. Human Reproduction $\mathbf{1 7}$ 2556-2559. (doi:10.1093/humrep/17.10.2556)

Shoji S, Yoshida N, Amanai M, Ohgishi M, Fukui T, Fujimoto S, Nakano Y, Kajikawa E \& Perry AC 2006 Mammalian Emi2 mediates cytostatic arrest and transduces the signal for meiotic exit via Cdc20. EMBO Journal 25 834-845.

Speirs S \& Kaufman MH 1990 Effect of maternal age on the incidence of digynic triploidy in LT/Sv strain mice: implications for the ovulation of primary and secondary oocytes in this strain. Journal of Experimental Zoology 253 83-87. (doi:10.1002/jez.1402530111) 
Steuerwald N, Cohen J, Herrera RJ, Sandalinas M \& Brenner CA 2001 Association between spindle assembly checkpoint expression and maternal age in human oocytes. Molecular Human Reproduction 7 49-55. (doi:10.1093/molehr/7.1.49)

Suzuki T, Suzuki E, Yoshida N, Kubo A, Li H, Okuda E, Amanai M \& Perry AC 2010 Mouse Emi2 as a distinctive regulatory hub in second meiotic metaphase. Development 137 3281-3291. (doi:10.1242/dev. 052480)

Tarkowski AK 1977 In vitro development of haploid mouse embryos produced by bisection of one-cell fertilized eggs. Journal of Embryology and Experimental Morphology 38 187-202.

Tsurumi C, Hoffmann S, Graeser R, Geley S \& Polanski Z 2004 The spindle assembly checkpoint is not essential for CSF arrest of mouse oocytes. Journal of Cell Biology 167 1037-1050. (doi:10.1083/jcb. 200405165)

Vogt E, Kirsch-Volders M, Parry J \& Eichenlaub-Ritter U 2008 Spindle formation, chromosome segregation and the spindle checkpoint in mammalian oocytes and susceptibility to meiotic error. Mutation Research 65 14-29. (doi:10.1016/j.mrgentox.2007.10.015)
Wang JZ, Sui HS, Miao DQ, Liu N, Zhou P, Ge L \& Tan JH 2009 Effects of heat stress during in vitro maturation on cytoplasmic versus nuclear components of mouse oocytes. Reproduction 137 181-189. (doi:10.1530/REP-08-0339)

Wei L, Liang XW, Zhang QH, Li M, Yuan J, Li S, Sun SC, Ouyang YC, Schatten H \& Sun QY 2010 BubR1 is a spindle assembly checkpoint protein regulating meiotic cell cycle progression of mouse oocyte. Cell Cycle 9 1112-1121. (doi:10.4161/cc.9.6.10957)

Windt ML, Coetzee K, Kruger TF, Marino H, Kitshoff MS \& Sousa M 2001 Ultrastructural evaluation of recurrent and in-vitro maturation resistant metaphase I arrested oocytes. Human Reproduction 16 2394-2398. (doi:10.1093/humrep/16.11.2394)

Received 27 September 2011

Revised manuscript received 15 June 2012

Accepted 26 June 2012 